Trauma Berufskrankh 2010 · 12[Suppl 3]:256-258 DOI 10.1007/s10039-010-1606-y

Online publiziert: 17. März 2010

(c) Springer-Verlag 2010

\author{
A. Schad \\ Berufsgenossenschaft der Bauwirtschaft, Bezirksverwaltung Böblingen
}

\title{
Anspruch und Wirklichkeit ambulanter Operationen
}

- Ist er insoweit disponibel und subjektiv?

medizinischen Tagung in Baden-Baden im Oktober 2009 wurden die ambulante Chirurgie bzw. stationsersetzende Leistungen im Bereich der Gesetzlichen Unfallversicherung (GUV) nach den Gesichtspunkten Anspruch und Wirklichkeit aus 3 Blickwinkeln betrachtet. Beim vorliegenden Beitrag handelt es sich um eine schriftliche Zusammenfassung des Vortrages, in dem die Sicht der Verwaltung zu dieser Thematik aufgezeigt wurde. Die jetzige Darstellung konzentriert sich auf die wesentlichen Gesichtspunkte dieser Perspektive.

Bei der zu thematisierenden vergleichenden Analyse von Anspruch und Wirklichkeit waren zunächst der Anspruch der Verwaltung herauszuarbeiten, danach die Kriterien und Parameter für die Bewertung der Wirklichkeit zu definieren und diese anhand dieser Werte festzustellen, um sie einem Soll-Ist-Vergleich zuzuführen. Das im Beitrag verwendete Zahlenmaterial entstammt den Gemeinschaftsstatistiken und Auswertungen der Deutschen Gesetzlichen Unfallversicherung e.V. (DGUV).

\section{Anspruch der Verwaltung}

Diesbezüglich sind folgende Fragen interessant:

- Wie stellt sich der Anspruch der Verwaltung dar?

- Worauf gründet der Anspruch der Verwaltung?

- Manifestiert sich der Anspruch der Verwaltung im Gutdünken des einzelnen Mitarbeiters?

\section{Gesetzliche Grundlagen}

Bei der Verwaltung, deren Anspruch zu ambulanter Chirurgie und stationsersetzenden Leistungen zu beschreiben ist, handelt es sich um Berufsgenossenschaften (BG) und Unfallkassen als Träger der GUV. Berufsgenossenschaften und Unfallkassen gehören gemäß dem der staatlichen Ordnung immanenten und sie tragenden Prinzip der Gewaltenteilung der Exekutive an. Ihre Aufgabe sind die Vollziehung und Erfüllung der ihr durch die Legislative übertragenen Aufgaben. Sie sind deshalb an Recht und Gesetz gebunden. Demzufolge muss sich der Anspruch der Verwaltung entweder direkt aus einer Norm ergeben oder sich zumindest aus einer solchen ableiten lassen. Ein explizit normierter Anspruch der Verwaltung in Bezug auf ambulante Chirurgie und stationsersetzende Leistungen wurde nicht kodifiziert. Der Anspruch der Verwaltung an das ambulante Operieren ist als Ableitung aus dem gesetzlichen Auftrag der Träger der GUV zu begreifen.

Das Recht der GUV ist im 7. Buch des Sozialgesetzbuches - SGB VII - niedergelegt. Die Aufgaben der GUV sind als Dreiklang zu verstehen,

- zuerst die Prävention,

- dann die Rehabilitation und

- danach die Entschädigung.

Nach $₫ 1$ Nr. 2 SGB VII ist es Aufgabe der Träger der GUV, nach Eintritt von Arbeitsunfällen und Berufskrankheiten die Gesundheit und die Leistungsfähigkeit der Versicherten mit allen geeigneten Mitteln wiederherzustellen. Dieser Satz definiert das oberste Prinzip der Rehabilitation in der GUV. Alle Maßnahmen der daran teilnehmenden Akteure haben der Erfüllung diese Ziels zu dienen. Auch die ambulante Chirurgie und stationsersetzende Leistungen müssen sich an diesem Ziel messen lassen. Die Rehabilitation in der GUV umfasst dabei das gesamte Spektrum von der Erstversorgung am Unfallort bis zur Teilhabe des Versicherten am Arbeitsleben oder am Leben in der Gemeinschaft. Die Verpflichtung der Verwaltung, eine optimale Rehabilitation sicherzustellen, ist von dem Gedanken getragen, dass eine solche den Umfang der beim Versicherten verbleibenden Unfallfolgen minimiert und zu geringeren Kompensationen führt.

Um den Anspruch der Verwaltung auf eine optimale Rehabilitation zu sichern, haben die Unfallversicherungsträger alle Maßnahmen zu treffen, durch die eine möglichst frühzeitig einsetzende und sachgemäße Heilbehandlung gewährleistet wird. Sie können hierbei die von den Ärzten und Krankenhäusern zu erfüllenden Voraussetzungen und zu übernehmenden Pflichten festlegen (Durchführung der Heilbehandlung; $\$ 34$ Abs. 1 SGB VII). Von der Möglichkeit der Gestaltung der Durchführung der Heilbehandlung haben die Träger der GUV im Interesse der Standardisierung einer qualitativ hochwertigen Heilbehandlung umfassend Gebrauch gemacht. So wurden durch den Vertrag gemäß $\$ 34$ Abs. 3 SGB VII (Vertrag Ärzte/Unfallversicherungsträger) u. a. das D-Arzt-Verfahren (D-Arzt: Durchgangsarzt), das H-Arzt- 
Verfahren (H-Arzt: Heilbehandlungsarzt) und das Verletzungsartenverfahren (VAV) implementiert. Darüber hinaus wurden auch Rehabilitationsverfahren wie die Erweiterte Ambulante Physiotherapie (EAP) oder die Berufsgenossenschaftliche Stationäre Weiterbehandlung (BGSW) konzipiert. All diese Verfahren fordern als Teilnahmevoraussetzung von den Leistungserbringern die Erfüllung persönlicher und sachlicher Qualifikationsmerkmale. Auf diese Weise lässt sich in der Fläche der vom Gesetzgeber normierte und von der Verwaltung umzusetzende Anspruch einer optimalen Rehabilitation sicherstellen.

\section{Ambulante Chirurgie und stationsersetzende Leistungen}

Mit Inkrafttreten der Grundsätze zum Ambulanten Operieren in der GUV zum 01.01.2005 ist das ambulante Operieren jetzt auch für die Allgemeinheit sichtbar zu einem Element der Rehabilitation in der GUV geworden. Aufgrund der nachhaltigen Weiterentwicklung des ambulanten Operierens in der Krankenversicherung und dem qualitätsgesicherten Fortschritt in der Ausführung tragen nun, so die Annahme der Träger der GUV, auch ambulante Operationen und stationsersetzende Leistungen zur Verwirklichung der Rehabilitation der Versicherten mit allen geeigneten Mitteln bei. Der Anspruch der GUV-Träger auf Etablierung des ambulanten Operierens in der GUV wurde hierbei mit Aspekten der Forderung und Förderung unterlegt. Zum einen wurde der Katalog der Gesetzlichen Krankenversicherung zu ambulant durchführbaren Operationen und stationsersetzenden Eingriffen ( $\$ 115 \mathrm{~b}$ SGB V, Stand 01.01.2004) auch in der GUV für anwendbar erklärt; dies bedeutet auch bei bestimmten Eingriffen den Vorrang der ambulanten Behandlung vor einer stationären Maßnahme. Zum anderen wurden durch Anpassung der UV-GOÄ (Gebührenordnung für Ärzte im Vertrag Ärzte/ Unfallversicherungsträger) und durch Einführung von Zuschlägen bei ambulanter Versorgung finanzielle Anreize gesetzt. Allerdings sind die ambulante Chirurgie und stationsersetzende Leistungen kein eigenständiges Verfahren der Reha- bilitation in der GUV; vielmehr müssen sie sich in die bisher bewährten Strukturen einfügen.

Das Interesse der Träger der GUV an der Durchführung ambulanter Operationen liegt auch darin begründet, dass bei einer im Ergebnis gleichwertigen, den gesetzlichen Zielen der Rehabilitation gerecht werdenden Maßnahme auch für die GUV der Grundsatz der Wirtschaftlichkeit und Sparsamkeit gilt ( $\$ 69$ SGB IV).

\section{Wirklichkeit}

Knapp 5 Jahre nach Inkrafttreten der Grundsätze zum Ambulanten Operieren in der GUV stellt sich die Frage, ob sich das ambulante Operieren in der GUV tatsächlich etabliert hat und ob dies unter Wahrung des für die Träger der GUV geltenden hohen Qualitätsstandards geschehen ist.

Wichtige Parameter für die Abbildung der Wirklichkeit sind:

- die Gesamtzahl der ambulant durchgeführten Operationen,

- die Verteilung der Operationen auf die jeweils durchführende Einrichtung,

- die Häufigkeit einzelner Operationen,

- Qualität und

- Kosten.

Seit dem Berichtsjahr 2007 erfassen die gewerblichen Berufsgenossenschaften im Rahmen des Gesamtstatistikdatensatzes ambulante Operationen. Im Jahr 2008 wurden auf Basis dieser Erhebung 17.818 ambulante Operationen dokumentiert. Die gemeldete Anzahl gibt jedoch die Realität nicht vollständig wieder. Aufgrund des Prozederes bei der Erfassung werden ambulante Operationen meist erst mit Eingang der Rechnung beim Unfallversicherungsträger dokumentiert, sodass insbesondere im 4. Quartal des Jahres durchgeführte ambulante Operationen im betreffenden Jahr noch nicht erfasst sind. Bei einer Extrapolation der Meldungen der ersten 9 Monate des Jahres 2008 auf das Gesamtjahr 2008 ergibt sich eine Anzahl von rund $21.000 \mathrm{im}$ Jahr 2008 durchgeführten ambulanten Operationen. Diese wurden zu fast $55 \%$ von niedergelassenen Ärzten vorgenommen, der Anteil der BG-Kliniken und BG-Sonderstatio-
Trauma Berufskrankh 2010 - 12[Suppl 3]:

256-258

DOI 10.1007/s10039-010-1606-y

(C) Springer-Verlag 2010

\section{A. Schad}

\section{Anspruch und Wirklichkeit ambulanter Operationen}

\section{Zusammenfassung}

Die hohe Qualität ambulant durchgeführter

Operationen macht diese zu einem wichtigen

Element der von der Gesetzlichen Unfallversicherung (GUV) getragenen Rehabilitation. Mit diesen Eingriffen werden die Unfallversicherungsträger ihrem gesetzlichen Auftrag, der Rehabilitation mit allen geeigneten Mitteln, gerecht. Die große Anzahl ambulant durchgeführter Operationen dokumentiert deren Etablierung in der GUV. Diese Tatsache ist aus Sicht der Träger der GUV, auch unter Kostengesichtspunkten, positiv zu bewerten.

\section{Schlüsselwörter}

Ambulante Chirurgie · Rehabilitation · Gesetzliche Unfallversicherung · Qualität · Kosten

\section{Demand and reality in outpatient surgery}

\section{Abstract}

The high quality of outpatient surgery has made it an important part of rehabilitation in the German statutory accident insurance. It enables the accident insurers to fulfill their statutory mandate, i.e., rehabilitation by all appropriate means. The great number of outpatient surgical procedures documents their establishment in the statutory accident insurance; from the accident insurer's perspective, this is seen positively, also in terms of costs.

\section{Keywords}

Outpatient surgery · Rehabilitation - Statutory accident insurance $\cdot$ Quality $\cdot$ Costs 


\begin{tabular}{|c|c|}
\hline Einrichtung & $\begin{array}{l}\text { Anteil } \\
\text { (\%) }\end{array}$ \\
\hline $\begin{array}{l}\text { Praxis eines niedergelassenen } \\
\text { D-Arztes }\end{array}$ & 47,11 \\
\hline $\begin{array}{l}\text { Praxis eines niedergelassenen } \\
\text { H-Arztes }\end{array}$ & 4,14 \\
\hline $\begin{array}{l}\text { Praxis eines sonstigen } \\
\text { niedergelassenen Arztes }\end{array}$ & 3,23 \\
\hline BG-Klinik & 0,86 \\
\hline BG-Sonderstation & 0,16 \\
\hline $\begin{array}{l}\text { Sonstige Krankenhäuser } \\
\text { mit Zulassung VAV }\end{array}$ & 27,63 \\
\hline $\begin{array}{l}\text { Krankenhäuser ohne Zulassung VAV, } \\
\text { D-Arzt }\end{array}$ & 7,60 \\
\hline Sonstige Klinik & 9,27 \\
\hline \multicolumn{2}{|c|}{$\begin{array}{l}\text { BG Berufsgenossenschaft, D-Arzt Durchgangsarzt, } \\
\text { H-Arzt Heilbehandlungsarzt, VAV Verletzungs- } \\
\text { artenverfahren }\end{array}$} \\
\hline
\end{tabular}

nen beträgt nur $1 \%$. Die detaillierte Darstellung findet sich in $\bullet$ Tab. 1.

Bei der Häufigkeit einzelner ambulant durchgeführter Operationen zeigt sich eine Fokussierung auf Metallentfernungen, Nahtversorgungen und arthroskopische Eingriffe. Die 1o häufigsten Operationen des Jahres 2008 sind in $\mathbf{0}$ Tab. 2 dargestellt.

Die statistische Erhebung der Qualität von ambulanten Operationen bzw. der Komplikationsrate ist diffizil und nur begrenzt valide. Als Kenngröße einer möglichen Komplikation wurde eine stationäre Aufnahme von Versicherten innerhalb von 2 Wochen nach erfolgter ambulanter Operation gewählt. Nach Auswertung dieses statistischen Parameters ergab sich eine Quote von etwa o,6\% der Fälle einer ambulanten Operation, in denen es möglicherweise zu Komplikationen gekommen ist.

Kosten für ambulante Operationen werden entsprechend den in der UVGOÄ fixierten Sätzen abgerechnet. Bei derselben Indikation resultieren für die Unfallversicherungsträger Kostenvorteile im Vergleich zu stationär durchgeführten Eingriffen, die nicht ganz einfach zu quantifizieren sind. Eine beispielhafte Vergleichsberechnung zwischen in VAVKrankenhäusern ambulant durchgeführten Operationen und einer fiktiv stationären Behandlung ergibt Kostenvorteile

Tab. 2 Art der ambulant durchgeführten Operationen UV-GOÄ

Anteil (\%)

2354 Entfernung einer Nagelung und/oder Drahtung und/oder Verschraubung 19,03 (mit Metallplatten) aus großen Röhrenknochen

2073 Sehnen-, Muskel- und/oder Fasziennaht - ggf. einschließlich Versorgung einer 13,45 frischen Wunde

2189 Resezierende arthroskopische Operation eines Gelenkes mit z. B. Entfernung $\quad 12,44$ oder Teilresektion eines Meniskus

2010 Entfernung eines tief sitzenden Fremdkörpers auf operativem Wege aus $\quad 6,87$ Weichteilen und/oder Knochen

2353 Entfernung einer Nagelung und/oder Drahtung und/oder Verschraubung aus 5,39 kleinen Röhrenknochen; auch Stellschraubenentfernung

2347 Nagelung und/oder Drahtung eines gebrochenen kleinen Röhrenknochens 3,12 (z. B. Mittelhand, Mittelfuß)

2065 Abtragung ausgedehnter Nekrosen im Hand- oder Fußbereich, je Sitzung $\quad 2,43$

2405 Entfernung eines Schleimbeutels 2,22

3300 Arthroskopie - ggf. mit Probeexzision - als diagnostische Maßnahme 2,11

2397 Operative Ausräumung eines ausgedehnten Hämatoms, als selbstständige $\quad$ 1,97 Leistung

UV-GOÄ Gebührenordnung für Ärzte im Vertrag Ärzte/Unfallversicherungsträger

zwischen 200,00 EUR und 1200,00 EUR je Behandlungsfall.

\section{Fazit}

Ambulante Chirurgie und stationsersetzende Leistungen haben sich $\mathrm{m}$. E. in der GUV als ein relevantes Element der Rehabilitation etabliert. Rund $21.000 \mathrm{im} \mathrm{Jahr}$ 2008 durchgeführte ambulante Operationen sprechen eine deutliche Sprache. Beachtlich ist hierbei auch die Verbreitung in der Fläche. Fast 55\% der ambulanten Operationen werden von niedergelassenen Ärzten durchgeführt. Aufgrund der hohen Qualität der ambulant durchgeführten Eingriffe sind diese ein wichtiges Element bei der Rehabilitation der Versicherten mit allen geeigneten Mitteln, dem Auftrag der Träger der GUV. Die Etablierung ambulanter Chirurgie und stationsersetzender Leistungen ist aus Sicht der Träger der GUV, auch unter Kostengesichtspunkten, positiv zu bewerten.

\section{Korrespondenzadresse}

\section{A. Schad}

Berufsgenossenschaft der Bauwirtschaft,

Bezirksverwaltung Böblingen,

Friedrich-Gerstlacher-Straße 15,

71032 Böblingen

Andreas.Schad@bgbau.de
Danksagung. Ich bedanke mich ausdrücklich bei Herrn Prof. Dr. Günter Rothe, der es mir durch seinen Einsatz möglich machte, auf die Auswertungen der ambulanten Operationen aus den Gemeinschaftsstatistiken des Jahres 2008 der DGUV zurückgreifen zu können.

Interessenkonflikt. Keine Angaben 